\title{
Diversidad de hongos micorrícicos arbusculares en maíz con cultivo de cobertura y biofertilizantes en Chiapas, México
}

\section{Diversity of arbuscular mycorrhizal fungi in maize with cover crops and biofertilizers in Chiapas, Mexico}

\author{
Yolanda del Carmen Pérez-Luna ${ }^{1}$, José David Álvarez-Solís² ${ }^{2}$ Jorge Mendoza-Vega², Juan Manuel \\ Pat-Fernández ${ }^{2}$, Regino Gómez-Álvarez ${ }^{2}$ \& Laura Cuevas ${ }^{3}$
}

${ }^{1}$ Universidad Politécnica de Chiapas, Calle Eduardo J. Selvas s/n y Av. Manuel de J. Cancino, Colonia Magisterial, C. P. 29082, Tuxtla Gutiérrez, Chiapas, México.

${ }^{2}$ El Colegio de la Frontera Sur (CONACYT), Carretera Panamericana y Periférico Sur s/n. C. P. 29290. San Cristóbal de Las Casas, Chiapas, México.

3Universidad Autónoma de Tlaxcala. km 10 Carretera Texmelucan-Ixtacuixtla, Tlaxcala 90122, Tlaxcala, México.

yluna18@yahoo.com.mx; dalvarez@ecosur.mx

\section{RESUMEN}

El presente trabajo tuvo como objetivo conocer la densidad, abundancia y diversidad de especies de hongos micorrízicos arbusculares (HMA) asociadas al cultivo de maíz (Zea mays L.) en parcelas que han sido manejadas con bajos ingresos de insumos externos y evaluar su respuesta a la aplicación de abono verde/cultivo de cobertura (AVCC) y biofertilizantes. El trabajo se realizó en siete parcelas de productores de maíz, de las cuales tres han sido manejadas con frijol nescafé (Mucuna deeringiana Merr.) como AVCC y cuatro sin dicho antecedente de manejo. En cada una de las parcelas se establecieron cuatro tratamientos de biofertilización: 1) inoculación con micorriza arbuscular, 2) aplicación de fertilizante orgánico foliar, 3) inoculación con micorriza + fertilizante orgánico foliar, y 4) testigo, los cuales se ordenaron bajo un diseño de bloques completos al azar con siete repeticiones. En total se identificaron 23 morfoespecies, de las cuales 14 se 1 levaron a nivel de especie, siendo Glomus y Acaulospora los géneros predominantes. El número de especies con AVCC superó en 50\% al obtenido sin AVCC. En las parcelas con AVCC se encontró el 91,3\% de morfoespecies de HMA, mientras que en las parcelas sin AVCC el 60,9\%. El porcentaje de colonización micorrízica no varió significativamente $(p=0,7630)$ entre parcelas con y sin AVCC, sin embargo el nivel de colonización fue más alto con inoculación de micorrizas $(86,6 \%)$ que en el testigo (71\%). Se concluye que el AVCC y la inoculación con micorrizas tuvieron un efecto positivo en la diversidad de especies de HMA y en la colonización de la raíz, respectivamente.

Palabras clave: Micorriza arbuscular, biodiversidad, Mucuna deeringiana, Zea mays, agricultura orgánica.

\begin{abstract}
The present work was conducted with the objective of know the density, abundance and diversity of arbuscular mycorrhizal fungi (AMF) associated with maize (Zea mays L.) in plots that have been managed with low incomes of external inputs green manure/cover crop (AVCC) and biofertilizers. The work was conducted in seven farmer plots with maize crop, of which three have been managed with nescafe bean (Mucuna deeringiana Merr.) as AVCC and four without such antecedent of managing. In each plot were established four treatments of biofertilization: 1) inoculation with AMF, 2) foliar application of organic fertilizer, 3) mycorrhizal inoculation with foliar organic fertilizer, and 4) control, which were ordered under a randomized complete block design with seven replications. In total we identified 23 morphospecies, 14 of which were carried at level of species, being Glomus and Acaulospora the dominant genera. The number of species in plots with AVCC was $50 \%$ higher than without AVCC. In the plots with AVCC was found $91.3 \%$ of morphospecies of AMF, while in plots without AVCC the $60.9 \%$. The percentage of mycorrhizal colonization was not significantly different $(\mathrm{p}=0.7630)$ between plots with and without AVCC, but the colonization level was higher with mycorrhizal inoculation (86.6\%) that in the control $(71 \%)$. We conclude that AVCC and inoculation with mycorrhizae had a positive effect on species diversity of AMF and root colonization, respectively.
\end{abstract}

KEYwORDS: Arbuscular mycorrhiza, biodiversity, Mucuna deeringiana, Zea mays, organic agriculture. 


\section{INTRODUCCIÓN}

La micorriza arbuscular (MA) es la simbiosis mutualista que se establece entre hongos del phylum Glomeromycota y la mayoría de plantas vasculares (Schübler et al. 2001), es de gran importancia en los sistemas agrícolas (Gosling et al. 2006), y tiene capacidad de incrementar la absorción de nutrientes poco móviles, principalmente fósforo (P) (Sanders \& Tinker 1971, Nakano et al. 2001). Además, la MA confiere a la planta otros beneficios, tales como: estimulación del crecimiento, resistencia al ataque de plagas y enfermedades, tolerancia a estrés hídrico, y contribuye a mejorar la estructura del suelo (Bethlenfalvay \& Linderman 1992).

La taxonomía de hongos micorrícicos arbusculares (HMA) se basa en caracteres discretos de la estructura subcelular de las esporas (Morton \& Bentivenga 1994). Schübler (2006) clasificó los HMA en 10 géneros con aproximadamente 193 especies descritas. A pesar que la información sobre diversidad de HMA en ecosistemas naturales y manejados es escasa, existen evidencias de que ésta podría ser mayor que la reportada (Opik et al. 2008). Borstler et al. (2006) estimaron que en el mundo podría haber 1250 especies de HMA. No obstante existe controversia de cómo cambia la diversidad de HMA en ecosistemas naturales que son transformados en agroecosistemas (Sieverding 1991). Hendrix et al. (1995) mencionaron que la composición de especies de HMA puede ser explicada en respuesta a cambios en la comunidad de plantas debido a la naturaleza obligada de los simbiontes.

En sistemas agrícolas, las prácticas que se realizan afectan a las poblaciones de HMA, la composición de especies y la colonización micorrízica (Kurle \& Pfleger 1994, Schalamuk et al. 2006), de manera que la diversidad de estos hongos puede sufrir alteraciones por el manejo agrícola intensivo (Oehl et al. 2004, Lovera \& Cuenca 2007). El manejo adecuado de rotaciones de cultivos micotróficos afecta positivamente la colonización y la producción de esporas (Bethlenfalvay 1991). El uso de leguminosas como abono verde/cultivo de cobertura (AVCC) protege al suelo y aporta nutrientes que contribuyen al crecimiento, desarrollo y rendimiento del maíz (Philip \& Gamboa 2003, AyalaSánchez et al. 2009), y podría incrementar el número de esporas de HMA, especialmente del género Glomus (Blanco \& Gutiérrez 1998). Otra estrategia para optimizar el uso de HMA en la agricultura es la inoculación de la semilla con cepas seleccionadas (Sieverding 1986), aun cuando puede presentarse la competencia entre cepas nativas e introducidas, alterando la acción de éstas (Allen \& Boosalis 1983), con sus posibles efectos sobre la diversidad de HMA presentes en el suelo.

El maíz (Zea mays L.) es la especie cultivada con más amplia distribución en México, en donde cumple una función de gran importancia en el suministro alimentario familiar y se encuentra muy ligada a la cosmovisión de los pueblos indígenas (Ayala-Sánchez et al. 2009). Esta especie ha sido catalogada como una planta micotrófica facultativa que responde a la presencia de HMA en suelos con bajo o moderado nivel de fertilidad (Gavito \& Varela 1995, Gavito \& Miller 1998); aunque también se ha observado que el grado de dependencia micotrófica en suelos con bajo nivel de fósforo varía ampliamente entre variedades de maíz (Khalil et al. 1994).

La importancia socio-cultural del cultivo de maíz y la difícil situación en la que se encuentra la agricultura campesina debido al incremento del costo de los fertilizantes, entre otros factores, plantea la necesidad de encontrar alternativas agroecológicas que mejoren la calidad del suelo, la producción del cultivo y la productividad del trabajo. En el estado de Chiapas, algunos campesinos están incorporando nuevas prácticas agrícolas que les permitan asegurar el suministro alimentario familiar, mejorar el ambiente ecológico y alcanzar un crecimiento económico. Entre los procesos de innovación agroecológica que están siendo impulsados en el cultivo de maíz se encuentra el manejo de AVCC, la aplicación de abonos orgánicos líquidos y la inoculación de micorrizas arbusculares. La comprensión del efecto de estas prácticas agronómicas sobre la riqueza de especies y la composición de la comunidad de HMA podría contribuir en la identificación de estrategias de manejo que optimicen los beneficios de la MA en la producción de este cultivo básico. Por lo tanto, el presente trabajo tuvo como objetivo conocer la densidad, abundancia y diversidad de especies de HMA asociadas al cultivo de maíz en parcelas que han sido manejadas con bajos ingresos de insumos externos y evaluar su respuesta a la aplicación de AVCC (Mucuna deeringiana Merr.) y biofertilizantes (micorrizas y fertilizante orgánico foliar).

\section{MATERIALES Y MÉTODOS}

El trabajo se realizó en el ciclo agrícola primavera-verano de 2009 bajo condiciones de campo en el Ejido La Bella Ilusión, Municipio de Maravilla Tenejapa, en el estado de Chiapas, México, el cual se encuentra ubicado a $16^{\circ} 08^{\prime} 20^{\prime \prime} \mathrm{N}$, $91^{\circ} 17^{\prime} 44^{\prime \prime} \mathrm{O}$, y a una altitud media de $400 \mathrm{msnm}$. El clima es Am(f) cálido húmedo con lluvias en verano y precipitación media anual de $2.220 \mathrm{~mm}$ (COMAR 2001). La vegetación natural corresponde a selva alta perennifolia, que se alterna con parches de vegetación secundaria (acahuales), pastizales, y cultivos anuales y perennes. Los suelos en la clasificación FAO/UNESCO corresponden a Leptosoles réndzico y lítico, Luvisol crómico y Acrisol pélico.

El estudio se condujo con la participación de un grupo de productores que trabajan bajo un esquema de agricultura orgánica desde hace 7 años aproximadamente. Dentro de las prácticas agrícolas que han incorporado se encuentra 
la siembra de frijol nescafé (Mucuna deeringiana Merr.) como AVCC, el cual una vez que ha alcanzado su máximo crecimiento se roza y pica, y se incorpora al suelo previo a la siembra del maíz. Asimismo, cuentan con un módulo de propagación de hongos micorrízicos de donde obtienen el biofertilizante (constituido por Glomus geosporum, $G$. intraradices, G. claroideum, Acaulospora morrowiae y A. scrobiculata) que agregan a la semilla de maíz previo a su siembra; y un módulo de producción de fertilizante orgánico foliar que ellos mismos elaboran mediante un proceso de fermentación anaeróbica a base de leche, estiércol, melaza, ceniza y hojas del frijol nescafé, el cual es aplicado por aspersión en dos o tres ocasiones durante el crecimiento vegetativo del maíz.

Se eligieron siete parcelas, de las cuales tres han sido manejadas con frijol nescafé y cuatro sin dicho antecedente de manejo. Las dimensiones de las parcelas fueron de aproximadamente $1.500 \mathrm{~m}^{2}$, en las que se sembraron variedades de maíz criollo obtenidas de cosechas anteriores. Cada una de las parcelas fue dividida en cuatro unidades experimentales en las que se aplicaron cuatro tratamientos: 1) inoculación con micorrizas arbusculares, 2) aplicación de fertilizante orgánico foliar, 3) inoculación con micorriza + fertilizante orgánico foliar, y 4) testigo, los cuales se ordenaron bajo un diseño de bloques completos al azar con siete repeticiones. Antes de la siembra se realizó un muestreo de suelos a $20 \mathrm{~cm}$ de profundidad con 15 sub-muestras que se mezclaron para formar una muestra compuesta en cada una de las parcelas para determinar sus propiedades físicas y químicas. Los suelos presentaron textura arcillosa: arena (22\%), limo (22\%) y arcilla (56\%); y sus principales atributos físicos y químicos se presentan en la Tabla I.

Las semillas de maíz fueron inoculadas con el biofertilizante micorrícico sólido producido por los mismos productores del ejido, el cual presentó 92,3 $\pm 45,6$ esporas $/ 10 \mathrm{~g}$ de sustrato y raíces finas con $84,0 \pm 9,6 \%$ de colonización micorrícica. Para la inoculación $1 \mathrm{~kg}$ de semillas de maíz se humedeció con aproximadamente $100 \mathrm{ml}$ de agua, éstas fueron peletizadas con $0,250 \mathrm{~kg}$ de inóculo micorrízico y se dejaron secar por toda una noche. Al siguiente día se sembraron en los tratamientos Micorriza y Micorriza+Foliar evitando la exposición directa al sol. El fertilizante foliar contenía $29,3 \%$ de materia orgánica, $2,07 \%$ de nitrógeno y $0,59 \%$ de fósforo. Este se aplicó en dos ocasiones con mochila tipo aspersor en dosis de $0,500 \mathrm{~L}$ para cada parcela.

Las parcelas tuvieron diferente composición y estructura vegetal (Tabla II), tales como: la presencia o no de sistemas agroforestales de maíz con árboles frutales y maderables, así como la asociación y rotación con cultivos de cobertura como frijol común (Phaseolus vulgaris), calabaza (Cucurbita sp.) y nescafé (Mucuna sp.).

Para la identificación morfológica de las especies de HMA, a los 90 días después de la siembra (dds) (10 de agosto de 2011) se recolectó una muestra compuesta de suelo rizosférico (formada por 15 submuestras) para cada uno de los tratamientos establecidos en cada parcela, así como de raíces finas de plantas de maíz en estadio de floración para determinar el porcentaje de colonización del HMA. Las raíces fueron aclaradas con $\mathrm{KOH} \mathrm{y} \mathrm{H}_{2} \mathrm{O}_{2}$, y teñidas con azul tripano en lactoglicerol (Phillips \& Hayman 1970). El porcentaje de colonización micorrízica se determinó en segmentos de raíces finas con un microscopio compuesto (100x) en tres campos visuales equidistantes sobre cada segmento y se registró la presencia o ausencia de estructuras micorrízicas como hifas, vesículas, arbúsculos y esporas (Giovannetti \& Mosse 1980). La extracción de esporas se realizó en muestras de $50 \mathrm{~g}$ de suelo rizosférico por el método de tamizado y decantación en húmedo con centrifugación en gradiente de sacarosa $(50 \% \mathrm{~m} / \mathrm{v})($ Gerdemann \& Nicolson 1963). Del total de esporas aisladas se tomó una muestra de 420 esporas, estas esporas fueron clasificadas por morfotipos de acuerdo a su tamaño y color con un microscopio estereoscópico; y fueron montadas en portaobjetos utilizando polivinil lactoglicerol (PVLG) y PVLG+reactivo Melzer (1:1 v/v) (Morton 1988) y observadas con un microscopio compuesto para diferenciar las características de sus paredes (grosor, color, ornamentaciones, reacción con Melzer), y de la hifa (presencia, forma, tamaño), colocándose 5 esporas para cada reactivo de montaje. La determinación taxonómica se realizó mediante el manual de Schenk \& Pérez (1990) y la consulta de la página de Internet de The International Culture Collection of Arbuscular Mycorrhizal Fungi - INVAM (http://invam.caf.wvu.edu).

La densidad de esporas se expresó como el número total de esporas presentes en $50 \mathrm{~g}$ de suelo rizosférico. La abundancia relativa se obtuvo como el número de esporas de un morfotipo o especie sobre el total de esporas aisladas multiplicado por 100. La riqueza se obtuvo como el número de especies, y para el cálculo del índice de diversidad, se consideró el método propuesto por Shannon-Weaver ( $\left.\mathrm{H}^{\prime}\right)$ (Shannon \& Weaver 1949) calculado con la siguiente fórmula: $H^{\prime}=-\sum p_{i} \ln p_{i}$, siendo $p_{i}$ el número de esporas de un morfotipo dividido entre el total de esporas aisladas.

Para evaluar el efecto del antecedente de cobertura (AVCC) y de la biofertilización sobre la densidad de esporas, la abundancia y diversidad de HMA, y el porcentaje de colonización micorrícica, se aplicó un análisis de varianza factorial con el antecedente de cobertura (parcelas con y sin AVCC) y tratamientos de biofertilización (Micorriza y Foliar) como factores principales, en el cual se incluyó la interacción Micorriza*Foliar. Se utilizó un nivel de probabilidad de 0.05 para definir los valores de significancia. Este análisis se realizó con el software InfoStat v. 2008 (Di Rienzo et al. 2008), mientras que para el índice de diversidad (índice de Shannon-Weaver $\left(\mathrm{H}^{\prime}\right)$ se utilizó el software FDiversity 2008 (Casanoves et al. 2008). Se utilizó la prueba de comparación de medias de Tukey $(\mathrm{p}<0,05)$. 
TABLA I. Características físicas y químicas del suelo en parcelas con y sin AVCC.

TABLE I. Soil physical and chemical characteristics in plots with and without AVCC.

\begin{tabular}{lccccccc}
\hline \multicolumn{1}{c}{ Parcela } & AVCC & $\begin{array}{c}\mathrm{DA}(\mathrm{g} / \\
\mathrm{ml})\end{array}$ & $\mathrm{pH}$ & $\begin{array}{c}\mathrm{MO} \\
(\%)\end{array}$ & $\begin{array}{c}\mathrm{N} \text { total } \\
(\%)\end{array}$ & $\mathrm{P}(\mathrm{mg} / \mathrm{kg})$ & $\begin{array}{c}\text { CIC }(\mathrm{cmol} / \\
\mathrm{kg})\end{array}$ \\
\hline El Cerro & No & 1,00 & 6,0 & 8,9 & 0,48 & 8,9 & 82,1 \\
El Aguacate & Sí & 0,94 & 6,4 & 7,6 & 0,41 & 12,7 & 83,1 \\
La Y & Sí & 0,93 & 6,3 & 6,6 & 0,33 & 5,6 & 79,2 \\
La Y (2) & No & 0,95 & 6,6 & 6,6 & 0,33 & 8,0 & 65,8 \\
Las Ruinas & Sí & 1,03 & 6,5 & 6,0 & 0,30 & 5,3 & 82,7 \\
La Curva & No & 0,95 & 6,5 & 11,9 & 0,63 & 11,2 & 81,5 \\
El Cedro & No & 0,97 & 6.3 & 9,6 & 0,55 & 16,6 & 81,2 \\
\hline
\end{tabular}

$\mathrm{AVCC}=\mathrm{Abono}$ verde/cultivo de cobertura, $\mathrm{DA}=$ densidad aparente (probeta), $\mathrm{MO}=$ materia orgánica (Walkley y Black), $\mathrm{N}$ total=nitrógeno total (microKjeldahl), $\mathrm{P}=$ fósforo extraíble (Olsen), $\mathrm{CIC}=$ capacidad de intercambio de cationes (acetato de amonio 1N, $\mathrm{pH} 7$ ).

TABLA II. Características de las parcelas.

TABLE II. Characteristics of plots.

\begin{tabular}{|c|c|c|c|c|c|c|c|}
\hline \multirow[t]{2}{*}{ COMPOSICIÓN } & \multicolumn{7}{|c|}{ PARCELAS } \\
\hline & El Cerro & El Aguacate & LA Y & LA Y (2) & LAS RUINAS & La Curva & El Cedro \\
\hline Variedad de maíz & Pinul & Jarocho & $\begin{array}{l}\text { Chucuy, } \\
\text { Jarocho }\end{array}$ & Tacsa & $\begin{array}{l}\text { Olotillo } \\
\text { blanco }\end{array}$ & $\begin{array}{l}\text { Chucuy, } \\
\text { Olotillo } \\
\text { blanco }\end{array}$ & Tuxpeño \\
\hline Años de descanso & 8 & 1 & No & 1 & No & 2 & $3-4$ \\
\hline Años en cultivo & 1 & 4 & 15 & 3 & 6 & 12 & 2 \\
\hline Otros cultivos & No & No & $\begin{array}{c}\text { Frijol, } \\
\text { calabaza }\end{array}$ & No & Frijol & $\begin{array}{l}\text { Plátano, } \\
\text { lima, toronja, } \\
\text { frijol, cedro }\end{array}$ & $\begin{array}{l}\text { Frijol, } \\
\text { cedro }\end{array}$ \\
\hline
\end{tabular}

\section{RESULTADOS}

COMPOSICIÓN TAXONÓMICA DE HONGOS MICORRÍZICOS ARBUSCULARES EN LAS PARCELAS ESTUDIADAS

En el conjunto de muestras de suelo rizosférico analizadas se identificaron 23 morfoespecies, de las cuales 14 se llevaron a nivel de especie (Tabla III, Fig. 1). Los géneros Glomus y Acaulospora fueronmásabundantes con 11 y 8 morfoespecies, respectivamente; mientras que Entrophospora, Gigaspora e Intraspora fueron menos comunes con sólo una especie cada uno (Fig. 1). Las especies con mayor dominancia relativa fueron G. geosporum, G. claroideum y A. scrobiculata $(30,0,26,2$ y $12,6 \%$ respectivamente).

La densidad de esporas de HMA varió de 45 a 181 (en promedio) por $50 \mathrm{~g}$ de suelo en las parcelas estudiadas. Es de hacer notar que la parcela "El Cerro" es un terreno que estuvo en descanso por 8 años, abriéndose de nuevo al cultivo este año, y presentó un alto número de esporas (131 esporas $/ 50 \mathrm{~g}$ de suelo, media de 4 repeticiones) comparado con las otras parcelas (45 a 121 esporas/50 g de suelo, media de 4 repeticiones) excepto con la parcela "El Aguacate" que tuvo el valor más alto (181 esporas/50 g de suelo, media de 4 repeticiones); sin embargo, en la parcela "El Cerro" el número de especies de HMA fue bajo ( 8 especies/50 g de suelo, total de 4 repeticiones) comparado con las parcelas "El Aguacate" $\mathrm{y}$ "La Y" que tuvieron antecedente de frijol nescafé y se han cultivado por 4 y 15 años consecutivos, respectivamente, las cuales presentaron el mayor número de especies (13 y 16 especies $/ 50 \mathrm{~g}$ de suelo, total de 4 repeticiones) de HMA. Por el contrario, la parcela "El Cedro" en donde se introdujeron plántulas de cedro presentó el menor número de esporas y de especies de HMA (45 esporas y 6 especies/50 g de suelo, media y total de 4 repeticiones) (Tabla IV).

EFECTO DE AVCC y BIOFERTILIZANTES SOBRE LA DENSIDAD, RIQUEZA Y DIVERSIDAD DE ESPECIES DE HMA Y COLONIZACIÓN DE LA RAÍZ

La densidad de esporas y el porcentaje de colonización micorrícica no variaron con significancia estadística entre parcelas con y $\sin \operatorname{AVCC}(\mathrm{p}=0,33$ y $\mathrm{p}=0,73)$; no obstante, 
se observó que el número de esporas fue cerca de $30 \%$ más alto en parcelas con AVCC. El AVCC tuvo un efecto positivo y altamente significativo $(\mathrm{p}<0,01)$ sobre la riqueza de especies y el índice de diversidad. El efecto de Micorriza fue significativo $(\mathrm{p}=0,043)$ sobre el porcentaje de colonización micorrícica y no tuvo una influencia significativa sobre el número de esporas, la riqueza de especies y el índice de diversidad. Se observó una interacción significativa $(\mathrm{p}=0,010)$ Micorriza*Foliar para el porcentaje de colonización micorrícica (Tabla V). El número de especies con AVCC superó en $50 \%$ al obtenido sin AVCC. En las parcelas con AVCC se encontró 91,3\% del total de las morfoespecies identificadas de HMA, mientras que en las parcelas sin AVCC el 60,9\%. El índice de diversidad en parcelas con AVCC fue $34,4 \%$ más alto que sin AVCC. El porcentaje de colonización micorrícica de maíz fue más alto con Micorriza (80.3\%) que sin Micorriza (73,3\%) (Tabla VI). La interacción Micorriza*Foliar se debió a la ausencia de efecto de la micorriza cuando se aplicó el abono orgánico foliar (Fig. 2). Es de hacer notar que E. infrequens, I. schenckii y aff. Glomus (Fig. 1p, r, i) fueron observados únicamente en parcelas con AVCC; mientras que G. verruculosum (Fig. $1 \mathrm{~g})$ se encontró en una parcela sin AVCC. G. claroideum, G. geosporum y A. scrobiculata (Fig. 1a, b, n) estuvieron presentes en los cuatro tratamientos de biofertilización evaluados de todos los sitios; sin embargo G. sinuosum y $G$. verruculosum (Fig. 1f, g) se observaron sólo en donde no se inoculó con HMA. Estudios adicionales son necesarios para confirmar si la introducción de cepas alóctonas desplaza a las cepas nativas.
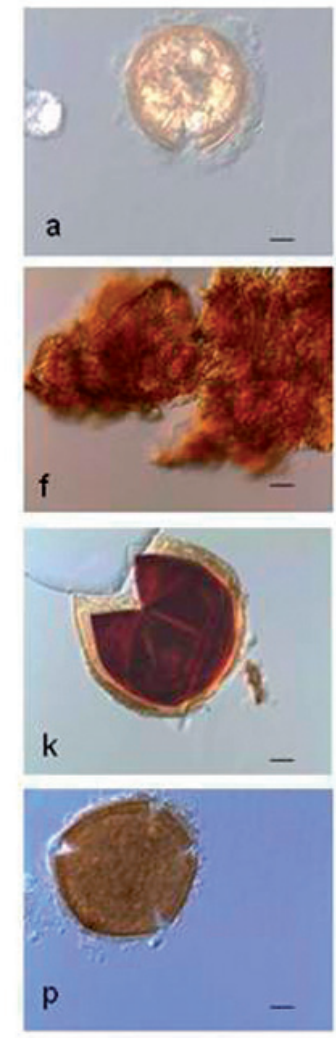
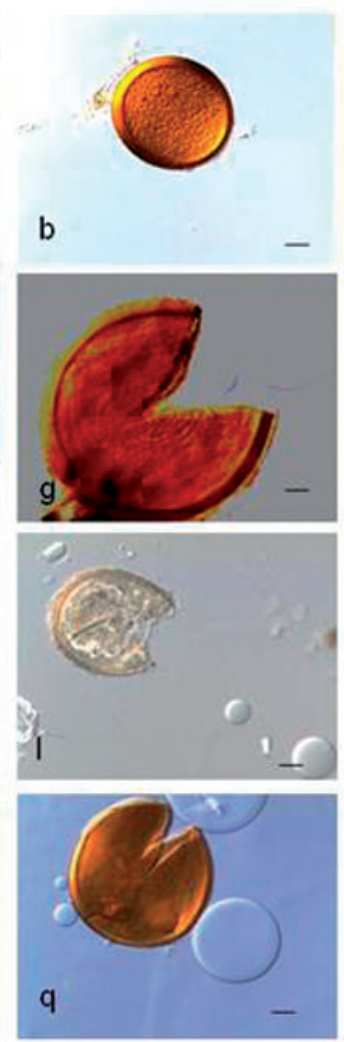
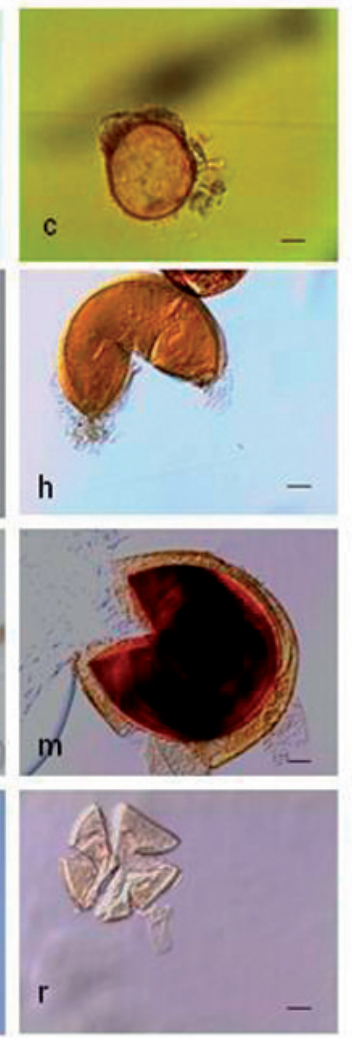
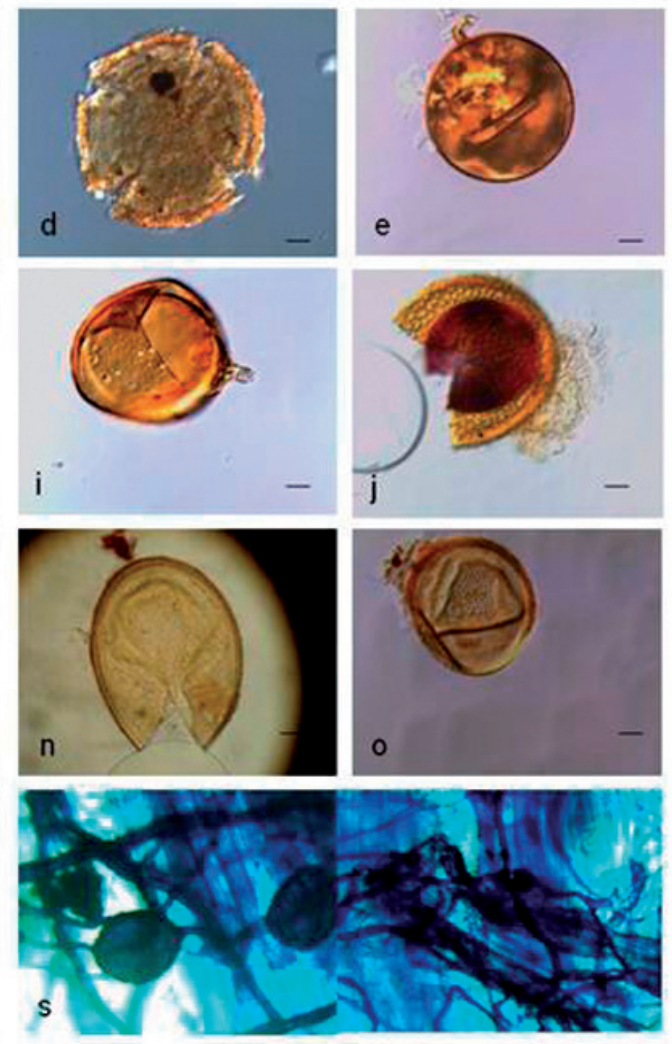

FIgURA 1. Especies y morfoespecies de hongos micorrízicos determinadas en las parcelas estudiadas del Ejido La Bella Ilusión, Maravilla Tenejapa, Chiapas. a) Glomus claroideum, b) G. geosporum, c) G. intraradices, d) G. aff. lamellosum, e) G. luteum, f) G. sinuosum, g) G. verruculosum, h) Glomus sp., i) aff. Glomus, j) Acaulospora excavata, k) A. mellea, 1) A. aff. morrowiae, m) A. spinosa, n) A. scrobiculata, o) A. aff. scrobiculata, p) E. infrequens, q) Gigaspora gigantea, r) Intraspora schenckii, s) Vesículas e hifas en raíces de maíz. Escala: 30 $\mu \mathrm{m}$.

FIGURE 1. Mycorrhizal fungi species and morphoespecies identified in studied plots of Ejido La Bella Ilusión, Maravilla Tenejapa, Chiapas. a) Glomus claroideum, b) G. geosporum, c) G. intraradices, d) G. aff. lamellosum, e) G. luteum, f) G. sinuosum, g) G. verruculosum, h) Glomus sp., i) aff. Glomus, j) Acaulospora excavata, k) A. mellea, 1) A. aff. morrowiae, m) A. spinosa, n) A. scrobiculata, o) A. aff. scrobiculata, p) E. infrequens, q) Gigaspora gigantea, r) Intraspora schenckii, s) Vesículas e hifas en raíces de maíz. $30 \mu \mathrm{m}$ scale. 


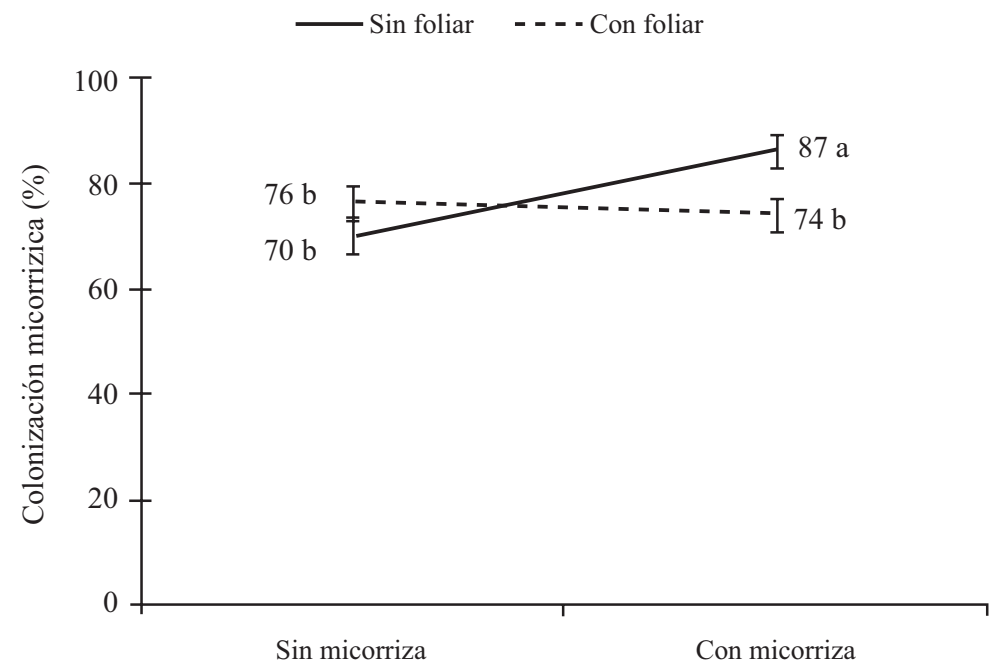

Figura 2. Efecto de la aplicación de micorrizas y fertilizante orgánico foliar sobre el porcentaje de colonización de la raíz de maíz. Las barras indican el error estándar de las medias $(n=7)$. Letras diferentes indican diferencias significativas (Tukey, $\mathrm{p}<0,05)$.

FIGURE 2. Effect of application of mycorrhizal and foliar organic fertilizer on percentage of root colonization of maize. Bars indicate standard error of the means $(n=7)$. Different letters indicate significant differences (Tukey, $\mathrm{p}<0.05$ ).

TABLA III. Especies y morfoespecies de HMA encontradas en la rizosfera de maíz.

TABLE III. AMF species and morphoespecies found in the rhizosphere of maize.

\begin{tabular}{|c|c|c|}
\hline ESPECIES Y MORFOESPECIES & $\begin{array}{c}\text { NÚMERO DE } \\
\text { ESPORAS }\end{array}$ & $\begin{array}{l}\text { ABUNDDANCIA } \\
\text { RELATIVA (\%) }\end{array}$ \\
\hline 1. Glomus claroideum N.C. Schenck \& G.S. Sm. & 110 & 26,2 \\
\hline 2. G. aff. claroideum N.C. Schenck \& G.S. Sm. & 2 & 0,5 \\
\hline 3. G. geosporum (T.H. Nicolson \& Gerd.) C. Walker & 126 & 30,0 \\
\hline 4. G. intraradices N.C. Schenck \& G.S. Sm. & 18 & 4,3 \\
\hline 5. G. aff. lamellosum Dalpé, Koske \& Tews & 6 & 1,4 \\
\hline 6. G. luteum L.J. Kenn., J.C. Stutz \& J.B. Morton & 27 & 6,4 \\
\hline 7. G. aff. luteum L.J. Kenn., J.C. Stutz \& J.B. Morton & 7 & 1,7 \\
\hline 8. G. sinuosum (Gerd. \& B.K. Bakshi) R.T. Almeida \& N.C. Schenck & 7 & 1,7 \\
\hline 9. G. verruculosum Blaszk. & 2 & 0,5 \\
\hline 10. G. sp. & 4 & 0,9 \\
\hline 11. Aff. Glomus & 5 & 1,2 \\
\hline 12. Acaulospora aff. denticulada Sieverd. \& S. Toro & 2 & 0,5 \\
\hline 13. A. excavata Ingleby \& C. Walker & 4 & 0,9 \\
\hline 14. A. mellea Spain \& N.C. Schenck & 5 & 1,2 \\
\hline 15. A. morrowiae Spain \& N.C. Schenck & 4 & 0,9 \\
\hline 16. A. aff. morrowiae Spain \& N.C. Schenck & 1 & 0,2 \\
\hline 17. A. spinosa C. Walker \& Trappe & 24 & 5,7 \\
\hline 18. A. scrobiculata Trappe & 53 & 12,6 \\
\hline 19. A. aff. scrobiculata Trappe & 2 & 0,5 \\
\hline 20. Entrophospora infrequens (I.R. Hall) R.N. Ames \& R.W. Schneid. & 3 & 0,7 \\
\hline 21. Gigaspora gigantea (T.H. Nicolson \& Gerd.) Gerd. \& Trappe & 4 & 0,9 \\
\hline 22. Intraspora schenckii (Sieverd. \& S. Toro) Oehl \& Sieverd. & 2 & 0,5 \\
\hline 23. I. aff. schenckii (Sieverd. \& S. Toro) Oehl \& Sieverd. & 2 & 0,5 \\
\hline TOTAL & 420 & 100 \\
\hline
\end{tabular}




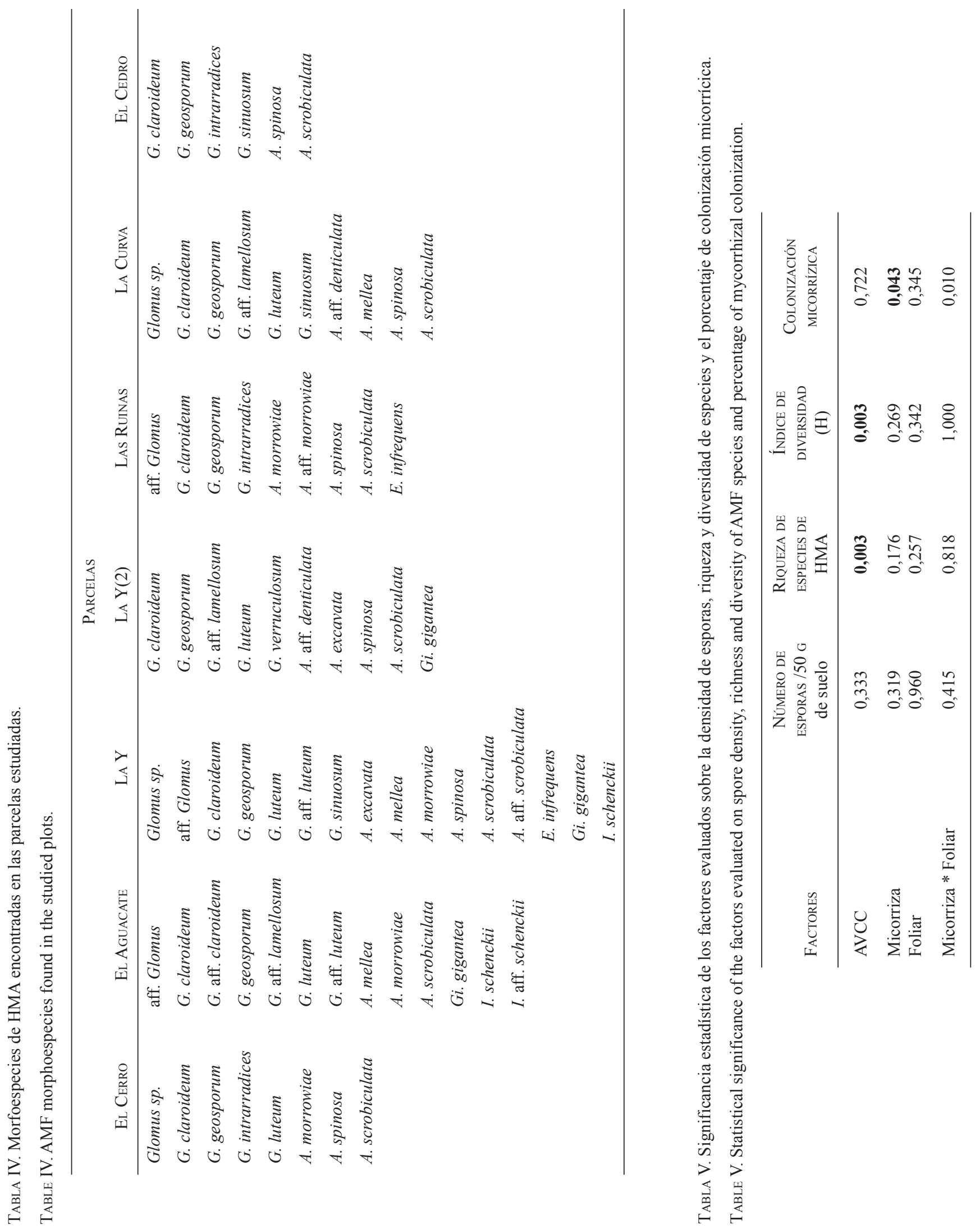


TABLA VI. Efecto de los factores evaluados sobre la densidad de esporas, riqueza y diversidad de especies de HMA y el porcentaje de colonización (valores medios \pm error estándar).

TABLE VI. Effect of the evaluated factors on spore density, richness and diversity of AMF species and percentage of mycorrhizal colonization (mean \pm standard error).

\begin{tabular}{|c|c|c|c|c|}
\hline FACTORES & $\begin{array}{l}\text { NÚMERO DE ESPORAS } \\
\text { /50 g DE SUELO }\end{array}$ & $\begin{array}{c}\text { RIQUEZA DE ESPECIES } \\
\text { DE HMA }\end{array}$ & $\begin{array}{l}\text { ÍNDICE DE DIVERSIDAD } \\
(\mathrm{H})\end{array}$ & $\begin{array}{c}\text { COLONIZACIÓN } \\
\text { MICORRÍZICA }\end{array}$ \\
\hline \multicolumn{5}{|c|}{ Abono verde } \\
\hline Sin & $94,6( \pm 18,57)^{\mathrm{a}^{*}}$ & $4,3( \pm 0,41)^{b}$ & $1,2( \pm 0,08)^{\mathrm{b}}$ & $77,4( \pm 2,16)^{\mathrm{a}}$ \\
\hline Con & $122,7( \pm 21,44)^{\mathrm{a}}$ & $6,3( \pm 0,47)^{\mathrm{a}}$ & $1,6( \pm 0,09)^{\mathrm{a}}$ & $76,3( \pm 2,49)^{\mathrm{a}}$ \\
\hline \multicolumn{5}{|l|}{ Micorriza } \\
\hline Sin & $94,4( \pm 19,96)^{\mathrm{a}}$ & $4,9( \pm 0,44)^{\mathrm{a}}$ & $1,4( \pm 0,09)^{\mathrm{a}}$ & $73,3( \pm 2,32)^{b}$ \\
\hline Con & $122,9( \pm 19,96)^{\mathrm{a}}$ & $5,7( \pm 0,44)^{\mathrm{a}}$ & $1,5( \pm 0,09)^{\mathrm{a}}$ & $80,3( \pm 2,32)^{\mathrm{a}}$ \\
\hline \multicolumn{5}{|l|}{ Foliar } \\
\hline $\operatorname{Sin}$ & $107,9( \pm 19,96)^{\mathrm{a}}$ & $5,6( \pm 0,44)^{\mathrm{a}}$ & $1,5( \pm 0,09)^{\mathrm{a}}$ & $78,4( \pm 2,32)^{a b}$ \\
\hline Con & $109,4( \pm 19,96)^{\mathrm{a}}$ & $4,9( \pm 0,44)^{\mathrm{a}}$ & $1,4( \pm 0,09)^{\mathrm{a}}$ & $75,3( \pm 2,2)^{\mathrm{ab}}$ \\
\hline
\end{tabular}

* Los valores con diferente letra, para efectos principales de cada factor, difieren significativamente $(\mathrm{p}<0,05)$.

\section{DISCUSIÓN}

En las parcelas estudiadas se encontraron 23 morfoespecies de HMA asociados a la rizosfera de maíz. El número de especies de HMA encontradas en este estudio es similar al reportado por Serralde \& Ramírez (2004) en suelos ácidos del Piedemonte Llanero en Colombia, quienes encontraron 24 morfotipos de HMA asociados a maíz fertilizado con abonos orgánicos (gallinaza) y abonos verdes (Caupí); Mathimaran et al. (2007) identificaron 18 especies de HMApertenecientes a los géneros Acaulospora, Gigaspora y Scutellospora en maíz con rotación de Crotalaria sp. cultivado en un suelo Ferralsol con mayor acidez $(\mathrm{pH}=5.0)$ que los suelos de las parcelas experimentales de este estudio. En otros estudios se registra un menor número de especies, tal es el caso de Carrenho et al. (2002) quienes registraron 14 especies de HMA en un experimento conducido en macetas de plástico con arena de cuarzo e inoculado con un concentrado de esporas de HMA que fue aislado del suelo de una parcela cultivada con maíz. Oehl et al. (2003) encontraron baja diversidad de HMA en monocultivos continuos de maíz con altos insumos, comparada con la observada en sitios cultivados por 7 años con rotación y aplicación moderada de insumos. Se estima que los monocultivos de maíz contienen entre 5 a 18 especies de HMA, lo cual está relacionado con diferentes prácticas agrícolas (Oehl et al. 2004, Jefwa et al. 2006).

El alto número de morfoespecies encontrado en el presente trabajo puede deberse al historial de manejo que han tenido estos terrenos, a una agricultura de bajos insumos y al momento en que se realizó la cuantificación de esporas. Al respecto, Oehl et al. (2004) encontraron que esporas de especies diferentes al género Glomus, como la mayoría de especies de Acaulospora, así como Entrophospora infrequens, estuvieron presentes únicamente en parcelas bajo el sistema de manejo orgánico y no bajo manejo convencional. Estos autores sugirieron que algunas especies de HMA, especialmente Acaulospora spp., encuentran un nicho ecológico en suelos de sistemas orgánicos y que puede estar relacionado con el bajo nivel de $\mathrm{P}$ disponible en esos suelos. Asimismo, se ha observado que en cultivos trampa bajo luz natural y temperatura ambiental se presenta una estacionalidad y/o patrón de sucesión en la esporulación de los HMA, las especies de Glomus son las primeras en esporular, mientras que especies de Acaulospora lo hacen después del periodo invernal y las especies de Scutellospora esporulan sólo entre octubre y diciembre, así como Gi. gigantea (Oehl et al. 2004).

La diversidad taxonómica es una medida de las especies de HMA presentes en el suelo, la cual puede ser afectada por las prácticas agrícolas así como por el cultivo de especies no micotróficas y el establecimiento de monocultivos, sin embargo la pérdida o disminución de la diversidad taxonómica tiene también un importante impacto sobre la diversidad funcional de los HMA (Sasvári et al. 2011).

Los índices de Shannon-Weaver obtenidos en las parcelas estudiadas $(1,22$ a 1,64$)$ son altos, comparados con los de Shannon-Wiener reportados por Serralde \& Ramírez (2004) en maíz bajo diferentes tratamientos (0,78 a 0.91$)$ y en diferentes años $(0,88$ a 1,01$)$ y a los reportados por Collins et al. (1991) en cultivos de maíz y soya (entre 0,42 y 1,59) para dos localidades de los Estados Unidos (Waseca y Lamberton).

El efecto positivo del AVCC sobre la riqueza de especies de HMA y el índice de diversidad, observado en este estudio, se debe principalmente a que éstos mejoran la 
actividad biológica del suelo (Crews \& Peoples 2004). Se ha reportado que el uso de leguminosas como cobertura, incrementa la población nativa de HMA de dos a tres veces (Deguchi et al. 2007) influyendo en la riqueza de especies y en su diversidad. Blanco \& Gutiérrez (1998) mencionan que Mucuna sp. incrementó las poblaciones de esporas del género Glomus principalmente. Sancho \& Cervantes (1996) reportan que Mucuna sp. al ser una planta altamente competitiva modifica la composición florística del agroecosistema, influyendo en la composición de especies de HMA (Blanco \& Gutiérrez 1998).

En los estudios sobre diversidad de HMA se pueden detectar especies dominantes o generalistas. Los resultados obtenidos parecen indicar que de todas las especies identificadas $G$. geosporum y $G$. claroideum parecen manifestarse como generalistas porque fueron encontradas en todas las parcelas evaluadas con una abundancia de 30,0 y $26,2 \%$, respectivamente, lo cual sugiere que pueden competir eficientemente y asegurar el éxito de la inoculación en campo; sin embargo, es importante considerar que la incorporación de inóculos conformados por diversas especies representan una ventaja funcional sobre las plantas cultivadas en sistemas agrícolas. Tal es el caso de la mayor colonización micorrízica observada en las plantas de maíz que fueron inoculadas con el biofertilizante. El género Glomus tiene una amplia distribución (Opik et al. 2006), por lo que se le ha considerado generalista (Oehl et al. 2003). La dominancia de Glomus en los suelos agrícolas estudiados se debe posiblemente a que cuenta con un micelio extrarradical altamente infectivo, mientras que otros géneros como Gigaspora se desarrollan frecuentemente a partir de esporas (Hart \& Reader 2002). En este trabajo Glomus fue el género predominante, lo cual concuerda con la literatura en donde se menciona que las especies que pertenecen a este género tienen un amplio rango de adaptación en cuanto al tipo de suelo y a las condiciones edafoclimáticas (Kahiluoto et al. 2001), además de ser altamente infectivo (Rivera et al. 2003); especies de Acaulospora prefieren suelos con $\mathrm{pH}$ bajos (Siqueira et al. 1984); por su parte, Entrophospora es altamente diverso en los trópicos (Sieverding 1991) mientras que Gigaspora es menos frecuente en agroecosistemas alterados (Siqueira et al. 1989) y algunas de sus especies se ven favorecidas en suelos arenosos; en este estudio estos dos últimos géneros, al igual que Intraspora, fueron los menos diversos.

La alta dominancia de G. claroideum observada en el presente trabajo coincide con los estudios realizados en agroecosistemas en Finlandia (Kahiluoto \& Vestberg 1999) y su amplia distribución en suelos cultivables de Europa y los Estados Unidos, en donde además se ha observado que forma micorrizas con un amplio rango de hospedantes (Walker \& Vestberg 1998). Así mismo, A. scrobiculata ha sido reportada en suelos con un rango amplio de niveles de fertilidad (Kahiluoto et al. 2001) y fue una de las especies con alta dominancia en las parcelas estudiadas.

Por su parte, $A$. excavata es una especie descrita recientemente (Ingleby et al. 1994); en México existen reportes que la ubican en agroecosistemas cafetaleros de Veracruz (Heredia \& Arias 2008). En este estudio se encontró en dos parcelas contrastantes, una con presencia de leguminosas como cobertura mientras que la otra es un monocultivo de maíz, en ambos sitios se observaron valores similares de $\mathrm{pH}(6,3-6,5), \mathrm{MO}(6,6-6,0), \mathrm{N}(0,33-0,30)$ y $\mathrm{P}(5,6-5,3)$. G. intraradices estuvo presente únicamente en tres sitios, aun cuando se esperaba que fuese una especie mucho más generalista; al respecto pudo haber influido que esta especie presenta esporas pequeñas que al momento de la extracción se hayan perdido.

La diversidad de HMA ha sido poco estudiada para el Estado de Chiapas, en este trabajo se identificaron 23 morfoespecies asociadas al cultivo de maíz, de las cuales únicamente dos habían sido reportadas en cultivos de caña de azúcar y coco (Varela \& Trejo 2001); y de las 14 especies de HMA registradas, 12 se encontraron en suelos que no fueron inoculados con el hongo micorrícico, tratándose por lo tanto de especies nativas.

\section{CONCLUSIONES}

Se identificaron 23 morfoespecies de HMA en la rizosfera de maíz, entre las cuales el género predominante fue Glomus, mostrando a su vez mayor diversidad (11 especies), seguido por Acaulospora (8 especies), Intraspora (2 especies), Entrophospora y Gigaspora (con un sola especie respectivamente). Glomus geosporum y Glomus claroideum fueron especies generalistas, y esta última se reconoce como especie dominante.

La presencia de cobertura de frijol nescafé afectó positivamente la riqueza y diversidad de HMA observadas en las parcelas de maíz, generando un índice de diversidad de 1,64 .

La biofertilización con micorrizas arbusculares incrementó el porcentaje de colonización de la raíz del maíz y no tuvo efecto significativo en la riqueza ni la diversidad de especies de HMA.

\section{AGRADECIMIENTOS}

Al FORDECYT/CONACYT por el apoyo financiero brindado para la realización de este trabajo, a través del proyecto No. 116306. A la alumna Jessica Ibeth Cruz López por su apoyo durante la identificación de especies de HMA. Al grupo de productores "La Ventana" del ejido La Bella Ilusión que hicieron posible este trabajo. A los revisores externos cuyos comentarios permitieron mejorar este documento. 


\section{BIBLIOGRAFÍA}

Allen, M. \& M. Boosalis. 1983. Effects of two species of VA mycorrhizal fungi on drought tolerance of winter wheat. New Phytologist 93: 67-76.

Ayala-SÁnchez, A., L. Krishnamurthy \& J.A. Basulto-Graniel. 2009. Leguminosas de cobertera para mejorar y sostener la productividad de maíz en el sur de Yucatán. Terra Latinoamericana 27: 63-69.

Bethlenfalvay, G.J. 1991. Mycorrhizae and crop productivity. In: G. J. Bethlenfalvay \& J. Linderman R. (eds), Micorrhizae in Sustainable Agriculture. pp. 1-27. Special Publication 54. American Society of Agronomy Madison, WI, U.S.A.

Bethlenffalvay, G.J. \& R.G. Linderman. 1992. Mycorrhizae in sustainable agriculture. ASA. Special Publication, Madison, Wisconsin. 124 pp.

Blanco, F. \& R. Gutiérrez. 1998. Efecto de la Mucuna sp. en la composición de la comunidad de hongos MA del suelo y en la respuesta del maíz a la inoculación con hongos MA. Agronomía Costarricense 22(2): 153-161.

Borstler, B., C. Renker, A. Kahmen \& F. Buscot. 2006. Species composition of arbuscular mycorrhizal fungi in two mountain meadows with differing management types and levels of plant biodiversity. Biology of Fertility and Soils 42: 286-298.

Carrenho, R., S.F.B. Trufem \& V.L.R. Bononi. 2002. Effects of using different host plants on the detected biodiversity of arbuscular mycorrhizal fungi from an agroecosystem. Revista Brasileira de Botánica 25(1): 95-101.

Casanoves, F., J.A. Di Rienzo \& L. Pla. 2008. User Manual FDiversity: Statistical software for the analysis of functional diversity. First Edition, Argentina. www. fdiversity.nucleodiversus.org.

Collins, N., F. Pfleger, R. Crookston, S. Simmons \& P. Copeland. 1991. Vesicular-Arbuscular mycorrhizas respond to corn and soybean cropping history. New Phytologist 117: 657663.

COMAR. 2001. Comisión Mexicana de Ayuda a Refugiados. Chiapas. URL: http://www.acnur.org/biblioteca/pdf/2086. pdf

Crews, T.E. \& M.B. Peoples. 2004. Legume versus fertilizer sources of nitrogen: ecological trade-offs and human needs. Agriculture, Ecosystems and Environment 102: 279-297.

Deguchi, S., S. Shimazaki, S. Uozumi, K. Tawaraya, H. Kawamoto \& O. TANAKA. 2007. White clover living mulch increases the yield of silage corn via arbuscular mycorrhizal fungus colonization. Plant and Soil 291(1): 219-229.

Di Rienzo, J.A., F. Casanoves, M.G. Balzarini, L. González, M. TABlada \& C.W. Robledo. 2008. InfoStat, versión 2008, Grupo InfoStat, FCA, Universidad Nacional de Córdoba, Argentina.

Gavito, M.E. \& L. Varela. 1995. Response of "criollo" maize to single and mixed species inocula of arbuscular mycorrhizal fungi. Plant and Soil 176: 101-105.

Gavito, M.E. \& M.H. Miller. 1998. Early phosphorus nutrition, mycorrhizae development, dry matter partitioning and yield of maize. Plant and Soil 199: 177-186.

Gerdemann, J.V. \& T.H. Nicolson. 1963. Spores of mycorrhizal Endogone species extracted from soil by wet sieving and decanting. Transactions of the British Mycological Society 46: 235-244.

Giovannetti, M. \& B. Mosse. 1980. An evaluation of techniques for measuring vesicular arbuscular mycorrhizal infection in roots. New Phytologist 84: 489-500.

Gosling, P., A. Hodge, G. Goodlass \& G.D. Bending. 2006. Arbuscular mycorrhizal fungi and organic farming. Agriculture, Ecosystems and Environment 113: 17-35.

Hart, M.H. \& R.J. ReAdER. 2002. Taxonomic basis for variation in the colonization strategy of arbuscular mycorrhizal fungi. New Phytologist 153: 335-344.

Hendrix, J.W., B.Z. Guo \& Z.-Q. An. 1995. Divergence of mycorrhizal fungal communities in crop production systems. In: H.P. Collins, G.P. Robertson \& M.J. Klug (eds), The significance and regulation of soil biodiversity, p. 131-140. Kluwer Academic Publishers. Netherlands.

Heredia A., G. \& R.M. Arias M. 2008. Hongos saprobios y endomicorrizógenos en suelos. En: R.H. Manson, V. Hernández-Ortiz, S. Gallina \& K. Mehltreter (eds.), Agroecosistemas cafetaleros de Veracruz. Biodiversidad, manejo y conservación, pp. 193-212. Instituto de Ecología A. C. Instituto Nacional de Ecología INE-SEMARNAT.

Ingleby, K., C. Walker \& P.A. Mason. 1994. Acaulospora excavata sp. nov. - an endomycorrhizal fungus from Cote d'Ivoire. Mycotaxon 50: 99-105.

INVAM (International Culture Collection of Vesicular Arbuscular Mycorrhizal Fungi). General life cycle, and the structures formed. URL: http://invam.caf.wvu.edu

Jefwa, J.M., R. Sinclair \& J.A. Maghembe. 2006. Diversity of glomales mycorrhizal fungi in maize/sesbania intercrops and maize monocrop systems in southern Malawi. Agroforestry Systems 67: 107-114.

Kahiluoto, H., E. Ketoja, M. Vestberg \& I. Saarela. 2001. Promotion of AM utilization through reduced $\mathrm{P}$ fertilization. 2. Field studies. Plant Soil 231: 65-79.

Kahiluoto, H. \& M. Vestberg. 1999. Impact of cropping system on mycorrhiza. In: J.E. Olesen, R. Eltun, M.J. Gooding, E.S. Jensen \& U. Kopke (eds.), Designing and testing crop rotations for organic farming, pp. 305-309. DARCOF Report no. 1.

Khalil, S., T.E. Loynachan \& M.A. Tabatabai. 1994. Mycorrhizal dependency and nutrient uptake by improved and unimproved corn and soybean cultivars. Agronomy Journal 86: 949-958.

Kurle, J.E \& F.L. Pfleger. 1994. The effects of cultural practices and pesticides on VAM fungi. In: F.L. Pfleger \& R.G. Linderman (eds.), Mycorrhizae and plant health. pp 101131. APS Press.

Lovera, M. \& G. Cuenca. 2007. Diversidad de hongos micorrízico arbusculares (HMA) y potencial micorrízico del suelo de una sabana natural y una sabana perturbada de la Gran Sabana, Venezuela. Interciencia 32(2): 108-114.

Mathimaran, N., R. Ruh, B. Jama, L. Verchot, E. Frossard \& J. JANSA. 2007. Impact of agricultural management on arbuscular mycorrhizal fungal communities in Kenyan ferralsol. Agriculture, Ecosystems and Environment 119: 22-32.

Morton, J.B. 1988. Taxonomy of VA mycorrhizal fungi: classification, nomenclature, and identification. Mycotaxon 32: $267-324$ 
Morton, J.B. \& S.P. Bentivenga. 1994. Levels of diversity in endomycorrhizal fungi (Glomales, Zygomycetes) and their role in defining taxonomic and non-taxonomic groups. Plant Soil 159: 47-59.

NaKano, A., T. KazUshi \& M. Kimura. 2001. Effect of host shoot clipping on carbon and nitrogen sources for arbuscular mycorrhizal fungi. Mycorriza 10(6): 287-293.

Oehl, F., E. Sieverding, K. Ineichen, P. Mäder, T. Boller \& A. WiemKen. 2003. Impact of land use intensity on the species diversity of arbuscular mycorrhizal fungi in agroecosystems of Central Europe. Applied and Environmental Microbiology 69: 2816-2824.

Oehl, F., E. Sieverding, P. Mäder, D. Dubois, K. Ineichen, T. Boller \& A. WiemKen. 2004. Impact of long-term conventional and organic farming on the diversity of arbuscular mycorrhizal fungi. Oecologia 138: 574-583.

OpiK, M., M. Moora, J. LiIra \& M. Zobel. 2006. Composition of root-colonizing arbuscular mycorrhizal fungal communities in different ecosystems around the globe. Journal of Ecology 94: 778-790.

Opik, M., M. Moora, M. Zobel, U. Saks, R. Wheatley, F. Wright \& T. DANiell. 2008. High diversity of arbuscular mycorrhizal fungi in a boreal herb-rich coniferous forest. New Phytologist 179(3): 867-876.

Philip, D. \& W. Gamboa. 2003. Observaciones sobre el sistema Mucuna-maíz en laderas de Waslala, Región Atlántica de Nicaragua. Agronomía Mesoamericana 14(2): 215-221.

Phillips, J.M. \& D.J. Hayman. 1970. Improved procedures for clearing and staining parasitic and vesicular-arbuscular mycorrhizal fungi for rapid assessment of infection. Transactions of the British Mycological Society 55: 158161.

Rivera, R., F. Fernández, A. Hernández, J.R. Martín \& K. FERNÁNDEZ. 2003. Bases científico-técnicas para el manejo de los sistemas agrícolas micorrizados eficientemente. En: R. Rivera, \& K. Fernández (eds.), Manejo efectivo de la simbiosis micorrízica, una vía hacia la agricultura sostenible. Estudio de caso: el Caribe. INCA. La Habana. $166 \mathrm{p}$.

Sancho, F. \& C. Cervantes. 1996. El uso de plantas de cobertura en sistemas de producción de cultivos perennes y anuales en Costa Rica. En: F. Bertsch, W. Badilla \& E. Bornemisza (eds.), Congreso Nacional Agronómico y de Recursos Naturales, Vol. 3, Suelos, p. 181-188. UNED y EUNA. San José, Costa Rica.

SAnders, F.E. \& P.B. Tinker. 1971. Mechanism of absorption of phosphate from soil by Endogone mycorrhizas. Nature 233: $278-279$

SASvÁRI, Z., L. HornoK \& K. Posta. 2011. The community structure of arbuscular mycorrhizal fungi in roots of maize grown in a 50-year monoculture. Biology and Fertility of Soils 47: 167-176.

Schalamuk, S., S. Velázquez, H. Chidichimo \& M. Cabello. 2006. Fungal spore Diversity of arbuscular mycorrhizal fungi associated with spring wheat: effects of tillage. Mycología 98(1): 16-22.

SCHENK, N.C. \& Y. PÉrez. 1990. Manual for the identification of VA Mycorrhizal Fungi. Synergistic Publications, Gainsville USA.

Schübler, A., D. Schwarzott \& C. Walker. 2001. A new fungal phylum, the Glomeromycota: phylogeny and evolution. Mycological Research 105: 1413-1421.

SchÜBler, A. 2006. Phylogeny and taxonomy of Glomeromycota ('arbuscular mycorrhizal (AM) and related fungi'). URL: http://AMF-phylogeny.com.

Serralde O., A.M. \& M.M. Ramírez G. 2004. Análisis de poblaciones de micorrizas en maíz (Zea mays) cultivado en suelos ácidos bajo diferentes tratamientos agronómicos. Revista Corpoica 5(1): 31-40.

Shannon, C.E. \& W. Weaver. 1949. The mathematical theory of communication. University of Illinois Press, Urbana, IL. EEUU. 144 pp.

Sieverding, E. 1986. El papel de las micorrizas en la agricultura. Suelos Ecuatoriales 16(1): 52-59.

SiEVERDING, E. 1991. Vesicular-arbuscular mycorrhiza management in tropical agrosystems. Eschborn, Germany: GT Z, 371 pp.

Siqueira, J.O., D.H. Hubbel \& A.W. Mahmud. 1984. Effects of lemming on spore germination, germ tube growth and root colonization by vesicular-arbuscular mycorrhizal fungi. Plant and Soil 76: 115-124.

Siqueira, J., A. Colozzi-Filho \& E. Olivei R. 1989. Ocurrencia de micorrizas vesículo arbusculares em agro ecossitemas naturais do estado de Minas Gerais. Pesquisa Agropecuaria Brasileira 24: 1499-1506.

VARela, L. \& D. TREJO. 2001. Los hongos micorrizógenos arbusculares como componentes de la biodiversidad del suelo en México. Acta Zoológica Mexicana 1(número especial): 39-51.

Walker, C. \& M. Vestberg. 1998. Synonymy amongst the arbuscular mycorrhizal fungi: Glomus claroideum, $G$. maculosum, G. multisubstenum y G. fistulosum. Annals of Botany 82: 601-624.

Recibido: 11.11.11

Aceptado: 29.02.12 